\title{
Understanding the Impact of Sustainability and CSR Information in D2C Online Shops on Consumer Attitudes and Behavior - A Literature Review
}

\author{
Maximilian Schacker \\ University of St.Gallen \\ maximilian.schacker@unisg.ch
}

\author{
Katarina Stanoevska-Slabeva \\ University of St.Gallen \\ katarina.stanoevska@unisg.ch
}

\begin{abstract}
Many brands nowadays use direct-to-consumer channels such as proprietary online shops, in order to provide information related to their sustainability and CSR initiatives and to increase consumers' perceptions of legitimacy of the company and its products. However, so far little is known about the effects of such information on consumer attitudes and behavior. This implies that the true benefit of S/CSR initiatives is currently not well understood by most companies and resource allocation in this area may be distorted. Therefore, in this literature review we consolidate and map existing research that can inform our understanding of this phenomenon. By analyzing a sample of 46 papers we find that research on the topic in a direct-to-consumer context is sparse, but that theories and empirical evidence from related contexts can help us grasp the issue to some extent.
\end{abstract}

\section{Introduction}

Sustainable consumption and production have been declared core sustainable development goals of the United Nations by its General Assembly in 2015 [1]. The rising awareness of the importance of sustainable consumption and production that is reflected in this decision presents an opportunity for many companies that embrace a sustainable business approach - not only in terms of cost savings (e.g. through higher energy efficiency) but also from a marketing perspective [2]. By portraying the firm and its products as environmentally friendly and socially responsible, companies aim to foster a positive brand image and to create additional customer value [3]. However, to reap the full benefits of their sustainability and corporate social responsibility (S/CSR) efforts, companies need to communicate these measures and their impact to their customers credibly and effectively [4].

Communicating S/CSR information to customers is a challenge for many organizations for two reasons: first, there is a fine line between transparently reporting S/CSR information and bragging about it. The latter can quickly lead to accusations of greenwashing and have adverse business effects (in addition to ethical concerns) [5]. Second, many companies are limited in the amount of, and control over S/CSR information that they can communicate over various touchpoints offered to their customers [6]. This is particularly true for consumer goods companies, which increasingly sell their products through online retailers. On digital retail platforms the freedom to provide company and product information varies. However, many platforms standardize product information to a high degree, so that the ability of any particular vendor to present S/CSR information is strongly confined $[6,7]$.

For companies that aim to differentiate themselves through sustainable practices, direct-to-consumer (D2C) selling without intermediation by a retailer presents an interesting alternative [7]. In recent years, eCommerce related technological advances and the rise of social commerce have made it significantly easier and more attractive for manufacturers of consumer goods to sell their products directly to their customers through an own online shop. Especially for startups, the barriers to entry for selling D2C are typically lower than for selling through a retailer [7]. As a result, socalled D2C brands like Warby Parker, Dollar Shave Club or Casper have emerged as earnest competitors for incumbent brands in many industries. Furthermore, many established companies like Nike or Ikea have set up their own branded online shops as an additional distribution channel with growing importance [7].

$\mathrm{S} / \mathrm{CSR}$ information is a very common theme in these shops [8, 9, 10]. For many D2C companies such as Bombas, Everlane, Patagonia, Toms Shoes or Warby Parker, positive environmental or social impact is a core value proposition and an integral part of their strategy [3]. However, the effect of such information on customer attitudes and behavior is not yet very well understood and a systematic review of the current state of research in this field does not exist to the best of the knowledge of the authors [11]. This is problematic for two reasons: first, the insufficient understanding of the phenomenon and the lack of design principles for $\mathrm{S} / \mathrm{CSR}$ information in $\mathrm{D} 2 \mathrm{C}$ online shops means that companies forego an opportunity to benefit fully from 
their S/CSR efforts. Second, it implies that the true impact of S/CSR activities on a company's bottom line will be hard to measure. Both these issues potentially distort companies' allocation of resources to S/CSR activities. This is not only problematic for the company but also constitutes a major roadblock on the way towards reaching the sustainable development goal of making production and consumption more sustainable.

Against this backdrop and as a foundation for future studies in this domain, this paper aims (1) to identify the most relevant theories that help to explain the effect of S/CSR information on consumer attitudes and behavior, (2) to map and synthesize existing empirical evidence on this phenomenon and (3) to identify and present the core practical and theoretical implications in the $\mathrm{D} 2 \mathrm{C}$ context.

\section{Conceptual Background}

\subsection{Sustainability vs. CSR Information}

Sustainability and CSR are two closely related and sometimes overlapping concepts. They both refer to "a more humane, more ethical, more transparent way of doing business" [12], yet they are not the same. A common notion of sustainability is based upon the United Nations' well-known definition of sustainable development: "meeting the needs of the present without compromising the ability of future generations to meet their own needs" [13]. Leaning on this definition and the discussion of sustainability and related terms by Van Marrewijk [12], we define sustainability in a corporate context as a mode of doing business that is economically viable while treating the environment and stakeholders in a way that affords a mutually beneficial coexistence. It is often linked to the idea of a triple bottom line which extends the concept of business success beyond the economic sphere and more holistically accounts for the dimensions people, planet and profit $[12,14]$.

The term CSR also reflects the notion that a company has a responsibility towards society beyond creating value for its shareholders. In its broadest sense it entails all actions of a company with net-positive effects on society or on the environment that the company operates in [15]. This may mean improving core elements of its business model, its products or its operations to make them more sustainable but could also include philanthropic actions like donating to charity or sponsoring events that are not directly related to the core business. These latter examples can benefit the public image of the company but may not necessarily be sustainable in a narrower sense.

While there are thus differences between sustainability and CSR, in practice these differences are often blurred. Companies tend to report on CSR and sustainability jointly or without differentiating between the two [12]. Therefore, we also decided to analyze the two concepts together in this study (using the acronym S/CSR).

\subsection{D2C Online Shops}

By D2C online shops we refer to websites controlled by a company through which it sells its own products directly to the end-customer. Typically, the term relates to companies that sell physical consumer goods and thus excludes providers of services and virtual items such as travel or event tickets and insurances as well as B2B companies. D2C online shops can be distinguished from corporate websites, which may list and describe products but do not sell them. They also differ from online retail platforms such as Amazon, which sell a large assortment of goods from various suppliers. A hybrid form are socalled digital "shop in shop" systems and social commerce sites where companies can install a branded presence within a larger marketplace [7]. In general, this comes with some freedom in designing various aspects of the branded page including the opportunity to provide S/CSR information. We thus include this hybrid form in our definition of D2C online shops.

As outlined in the introduction, D2C online business is on the rise [16]. According to a study by eMarketer, D2C eCommerce sales in the United States more than doubled between 2017 and 2019 from 6.85 billion US dollars to 14.28 billion US dollars and they are forecasted to keep growing at a high pace, reaching 21.25 billion US dollars in 2021 [17]. While this is still a modest volume compared to the total eCommerce market, the number of businesses involved in this segment is considerable (as most D2C companies are relatively small). The results of our research are thus potentially relevant to a growing audience of D2C brand companies and entrepreneurs.

\section{Literature Search and Review Method}

Methodologically, our review is based on the seminal works of Webster and Watson [18] as well as vom Brocke et al. [19, 20]. To ensure reproducibility and transparency, this section details our literature search and selection process as well as our approach to analyzing the identified literature.

\subsection{Paper Search and Selection}

As depicted in Figure 1, our literature search process consisted of a sequence of several steps: first, 


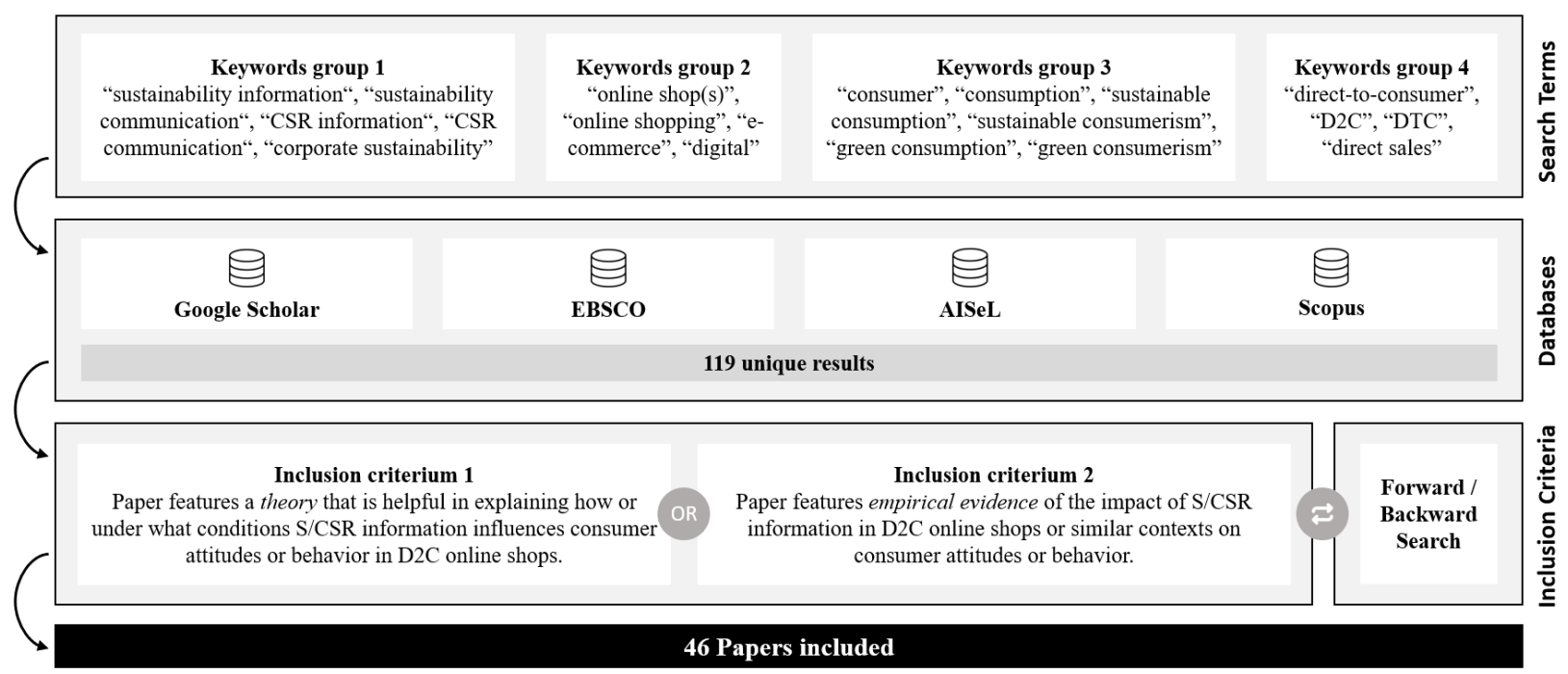

Figure 1: Literature Search Process

we selected relevant search terms that reflect key elements of the defined scope of this review. We divided them into four groups: group 1 contains keywords relating to S/CSR information, group 2 features search terms relating to the online shopping environment, group 3 focuses on consumer behavior and the final group accounts for the D2C context. Based on these groups we devised search queries that were subsequently entered in the respective database search interfaces. We used Google Scholar, EBSCO, AISeL and Scopus as our databases of choice, due to their interdisciplinary nature. This approach was chosen over a purely journal-based approach, because the topic touches upon different research streams so that relevant papers are dispersed across various publication outlets, which makes it difficult to pick a definite selection of relevant journals.

As a strict combination of all four keyword groups using an AND operator did not yield any results in any of the databases, we used different combinations of two or three of the keyword groups to obtain results that would match our specific research interest in most dimensions. We scanned the titles (and where necessary abstracts) of the search results and selected all those that matched at least two of our dimensions prima facie. We included peer-reviewed journal articles and conference proceedings, both theoretical and empirical. We ended up with 119 unique results after the initial screening.

Next, we devised two inclusion criteria. At least one of them had to be fulfilled for the paper to be included in our review. As the number of results matching our research focus exactly was sparse, we decided to include papers that could inform our research interest (1) by featuring a theory relevant to our case or (2) featuring empirical data on the impact of S/CSR information, even if collected in a different context. We also made sure not to include different versions (e.g. conference proceeding and journal publication) of the same paper. Using these filters, we reduced the number of eligible results to 37 . Finally, we used forward and backward search and identified 9 additional relevant articles, so that our final literature sample included 46 papers.

\subsection{Paper Analysis}

Each of the papers was read and coded by two reviewers. We extracted various data points from each paper including core themes, concepts and methods, but a particular focus was on theories and empirical research outcomes. An iterative approach in coding and inter-coder discussion were used to ensure stable, valid and reproducible results and a reasonable degree of objectivity.

\section{Results}

To give an insightful and structured account of our findings, the presentation of our results is divided into three parts: chapter 4.1. gives a short descriptive summary of the analyzed literature as a general overview and orientation. Chapter 4.2. presents the main theoretical lenses used in our literature sample to explain or predict the effect of S/CSR information on consumer behavior. The final part of this chapter summarizes and synthesizes core empirical research outcomes from the analyzed literature.

\subsection{Descriptive Summary}

Of the 46 papers included in our literature review, 18 were published in business publications (including 
marketing, accounting and corporate communications), 15 in S/CSR related publications, 10 in information systems and 3 in psychology publications. 37 of them are journal articles and 9 (all but one of them IS publications) are conference proceedings. The articles were crafted by 129 different authors in total.

\subsection{Core Theories}

In our literature sample, a variety of theories are used to inform our understanding of the influence of S/CSR information on consumer behavior. To present their core messages in a stringent and logical way we divided them into three groups: general consumer choice theories, communication and attribution theories and S/CSR specific theories.

4.2.1. General Consumer Choice Theories. As the outcome of interest in our research endeavor is consumer behavior, it is not surprising that a range of general consumer choice theories are deployed in our sample of papers. A foundational theory in this context is rational choice theory $[11,21,22]$. It predicts that consumers will act in a way that maximizes their own utility. Regarding their reaction to S/CSR information, this would imply that consumers care very little about it, or only where it affects them personally. This is indeed consistent with the finding that consumers react stronger to S/CSR messages that are also quality signals and could affect their personal health, such as an organic origin of product ingredients [11].

Another theory that is used in the identified articles and assumes rational consumers is game theory. Sachdeva et al. [23] and Schuitema and de Groot [21] (implicitly) use this theory as they model green consumerism as a social dilemma "in which consumers experience a conflict between their (short term) individual interests and (long term) collective interests". Each individual consumer will have the highest pay-off when acting selfishly, but if all consumers follow their self-interest, all consumers will be worse off. Only if all consumers follow the collective interests, everybody will be better off in the long term (however this equilibrium is hard to reach under the assumption of full rationality). This theory thus helps us to understand and appreciate why promoting sustainable consumption is so difficult. In its classic, hyper-rational form it would promote a skeptic view of the impact that S/CSR information can have on consumer behavior.

While there is some merit in these theories, it is acknowledged in many papers that consumers do not always act fully rational and selfish. Henkel et al. [24], for example, build upon the theory of bounded rationality, which suggests that rationality is limited by cognitive limitations of the mind and the time available to make a decision [25]. Rather than maximizing their utility, people use heuristics to choose a satisfactory solution to their decision problem. In a similar vein, Watts [26] uses dual-process theory to show how heuristic cues in sustainability rankings can influence user behavior. The theory posits that individuals make decisions using two different but connected mechanisms: an analytical and an intuitive one. She develops design principles for sustainability rankings (which may also be applied to a D2C context) that use heuristic cues and thus use the intuitive system to make S/CSR information more effective.

Nudging theory develops this idea further and suggests that consumer behavior can be altered in a predictable way through nudges, which are essentially modifications in the choice architecture that do not entail forbidding any options or significantly changing the economic incentives of the decision-maker [27]. In a digital context, a nudge is "a subtle form of using design, information and interaction elements to guide user behavior in digital environments, without restricting the individual's freedom of choice" [28]. This raises the question to what extent and in which presentation forms S/CSR information could be regarded as a nudge and which nudging techniques could be used to amplify its effectiveness in prompting sustainable consumption choices.

Another popular theory deployed by many papers in our sample is the theory of planned behavior [29, $30,31,32,33]$. It states that "behavior results from behavioral intention, which in turn is influenced by (1) attitude, (2) subjective norm (i.e. perceived social pressure), and (3) perceived behavioral control" [29]. As outlined by Zhang [29], there are challenges and implications in each of these three antecedents of behavioral intention in regard to the formation of sustainable consumption decisions: (1) consumers "tend to have difficulty in forming a specific attitude toward certain consumption behavior when they are not aware of the impact of a specific consumption decision on the environment" or when "they have difficulty in evaluating the impact of the specific consumption decision on the environment" [29]. Thus, S/CSR information at the point of sale may help consumers to form qualified opinions or attitudes about purchase decisions more easily. Regarding subjective norms (2), people will be more likely to form positive attitudes towards sustainable products if people close to them ("relevant others") also care strongly about S/CSR. Finally, perceived behavioral control (3) in our context can be translated as the degree to which the consumer trusts provided S/CSR information and feels that his or her purchase decision can make a difference [29, 30]. 
While the theory of planned behavior assumes that a behavioral intent will eventually result in the corresponding behavior, the so-called attitude-behavior gap, which is referred to multiple times in our literature sample [3, 8, 11, 29, 34, 35, 36, 37, 38], calls this link into question. Rather than a theory, the attitude-behavior gap is an empirical observation that there is a significant difference between what consumers say about their sustainability-related preferences and what they do. As the number of mentions of this phenomenon shows, it is a very common and important problem in sustainable consumption research and prompts us to be wary when drawing conclusions about consumer behavior from mere statements of intent.

4.2.2. Communication and Attribution Theories. The sender-receiver model developed by Kotler et al. [39] (though not a theory in a strict sense) is an important foundation for our understanding of communication processes in general and sustainability communication in specific. In this model, there are two main agents: the sender (in our case a D2C brand) and the receiver (the online shop customer). The sender needs to get a message (about S/CSR properties of the company or product) across to the receiver by means of a medium (the online shop website). The message is encoded by the sender and later decoded by the receiver. The message triggers a response (e.g. a purchase decision) from the receiver which is received as feedback by the sender. Finally, there is an element of noise consisting of random and rival messages, which could impair the communication [39]. Strähle et al. [8] adopt and modify this model to explain the effectiveness of the communication of sustainability in European fashion online shops and demonstrate that there are several break-up points which could prevent the message from triggering the desired response. They model greenwashing behavior as noise as it distorts the communication of true S/CSR efforts.

With this conceptual model of the communication process in mind, we can turn to other communication theories that link characteristics of the various model elements to communication effectiveness. For example, several papers refer to congruency theory, which posits that a high degree of fit between characteristics of the sender and the message facilitates easier processing of the message and reduces cognitive elaboration [4, 40, 41, 42, 43]. A S/CSR message that has a logical connection to the company's core business will be perceived as more congruent. However, the effect of congruency on the receiver's response seems to be ambiguous. Some papers conclude that harmonious (i.e. high fit) messages increase message credibility and will thus result in more sustainable behavior [4, 40, 43]. Others suggest that the relationship is not that clear. Becker-Olsen et al. [42], for example, elaborate that schema congruity theory would "predict an inverted-U shaped relationship, with moderate incongruity preferred to high or low congruity by consumers". They also introduce attribution theory according to which high levels of congruency may lead to dilution effects as consumers attribute firms' actions to self-interested motives.

Attribution theory is also used by Dunn and Harness [5] to explain how consumers evaluate S/CSR messages in social media. In general, the theory deals with how the message receiver uses information to arrive at causal inferences. In our case, it suggests that "favorability towards a CSR initiative depends on the attributions consumers develop towards the organization's motives for CSR" [5].

Another decisive factor of communication effectiveness is described by media richness theory [44]. It suggests that the medium must match the message, i.e. more complex messages require a richer medium. For S/CSR communication in online shops this entails that it is more effective when presented using rich media such as pictures, audio or video, rather than just text [44].

4.2.3. S/CSR Theories. While the theories introduced so far, are largely fundamental theories of psychology, marketing, communication or economics, that have been applied to the S/CSR communication context, some authors use more tailored theories, that have been developed specifically for the S/CSR context (some of which build upon the aforementioned theories).

An influential framework in this field is the CSR communication model developed by $\mathrm{Du}$ et al. [43]. It connects characteristics of the message (message content and channel) to internal and external communication outcomes with stakeholder and company characteristics as contingency factors. Like the sender-receiver model, it gives structure to S/CSR research but does not constitute a theory as such.

In contrast, goal framing theory fulfills the definition criteria of a theory. It states that even though people can have multiple goals at the same time, only the active or focal goal is the one that people act upon [45]. According to Schuitema and de Groot [21], "this explains why consumers can act more strongly on egoistic motives than on pro-social motives, even if they believe that collective interests are important".

Another theory that aims to explain the previously mentioned attitude-behavior gap in environmental decisions is neutralization theory. It suggests that consumers tend to use cognitive rationalization strategies to justify their unethical purchasing behavior as acceptable and to minimize remorse [38]. This could 
at least partially explain the absence of cognitive dissonance when consumers act against their ethical intentions. It implies that the effect of S/CSR information will depend on whether it helps to rationalize unethical behavior or whether it deters unsustainable purchasing decisions by creating larger cognitive dissonance.

Stern's value-belief-norm (VBN) theory was developed with the goal of crafting a "coherent theory of environmentally significant behavior" [46]. It states that the convergence of a person's values, beliefs, and personal norms drives his or her environmental behavior and incorporates elements of value theory, norm-activation theory and the new environmental paradigm [47]. The theory makes two important predictions about the impact of S/CSR information. First, some people (those who score high in altruistic and biospheric and low in egoistic values) are more likely to be influenced by S/CSR information than others based on their (relatively stable) value system. Second, the link from values to environmentalism is mediated by beliefs. Therefore, "environmentalist personal norms and the predisposition to proenvironmental action can be influenced by [S/CSR] information that shapes these beliefs".

4.2.4. Further Theories. Further theories and models that have been used in our literature sample to explain the role of S/CSR information in forming consumer behavior include contingency theory [48], legitimacy theory $[41,49,50]$, focus theory [51], framing theory [50], the SOR model [52], stakeholder theory [53], the theory of conspicuous consumption [54] and the theory of ethical egoism [53]. Unfortunately, discussing all of them in detail is beyond the scope of this review.

\subsection{Empirical Evidence}

Having discussed the major theories used in our literature sample to inform our understanding of the role of S/CSR information in shaping consumer behavior, we now turn to empirical evidence that has been collected on this issue so far. As mentioned earlier, we did not find any papers that analyze the impact of S/CSR information in the exact context that triggered our interest in this issue (i.e. D2C online shops). However, there is plenty of research that analyzes the same phenomenon in a slightly different context. While the results of this research are not a priori transferable to a D2C setting, they can help us devise informed hypotheses for this context. Also, some of the research designs used in these studies could be applied in a D2C context as well. Table 1 plots the research topics of the various papers and the context or medium in which they were explored. As can be seen from the table, empirical studies focus on message characteristics and how their effect on consumer attitudes and behavior is moderated by audience characteristics. Frequent research contexts are corporate websites and social media. Some studies do not specify the research context or just report that they conducted a survey presenting various types of S/CSR information, so that it is not clear for which context the setting is most representative. These papers are listed in the ultimate column in Table 1. While we cannot document all the findings within the scope of this paper, in the following we will present and synthesize the most important ones.

4.3.1. Message Characteristics. In general, it can be said that the evidence regarding the impact of S/CSR information on consumer decision making is mixed: some papers find a positive impact, some find no effect and some even report a negative impact on attitudes and purchase decisions under specific circumstances.

Various message characteristics play a key role in determining how effective the S/CSR communication is. In particular, the magnitude of reported S/CSR impact, the perceived issue importance, the use of ecolabels and the use of visual cues in general tend to have a positive impact on the effectiveness of S/CSR communication $[15,40,55,56,57]$. In addition, Amatulli et al. [54] report that information on external S/CSR activities (i.e. activities that are highly visible such as philanthropic projects) has a larger positive impact on brand perceptions than information on internal ones. The influence of the S/CSR issue (i.e. whether it is related to social, environmental, health or other causes) is not conclusive: while both environmental and social scores can have a positive effect according to some studies [15, 40, 55], O'Rourke and Ringer [11] report that information on these issues did not have a significant effect when displayed on a product review portal (in contrast to health information which had a positive effect). They even concede that environmental scores have a negative correlation with purchase intentions for some product categories, indicating an existing bias against "green" products.

The effect of perceived S/CSR fit (i.e. how well the S/CSR cause fits the company image) is equally ambiguous. Pérez et al. [40] show that higher perceptions of fit are only associated with higher consumer advocacy but not with higher purchase outcomes. Becker-Olsen et al. [42] find that low-fit initiatives as well as high-fit initiatives that are perceived to be profit-motivated negatively impact consumer beliefs, attitudes, and intentions, and Song and Wen [41] conclude that the effect of S/CSR fit is strongly moderated by controversiality of the industry. 


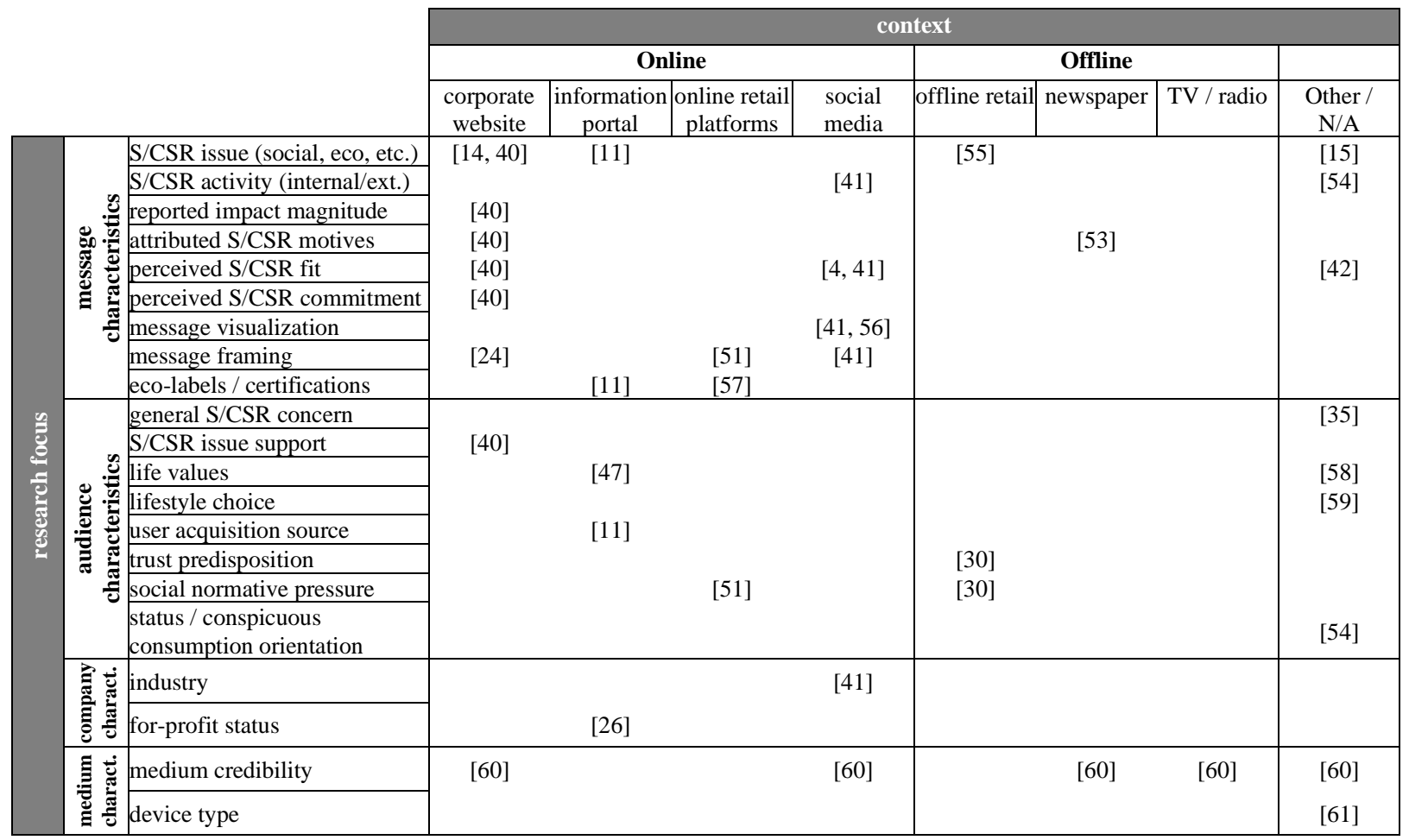

Table 1: Research topics and the context in which they were analyzed

4.3.2. Audience Characteristics. As theorized in $\mathrm{Du}$ et al.'s [43] CSR communication model, audience characteristics are frequently analyzed as a moderator of S/CSR communication effectiveness. The key insight from this research is that a general concern for S/CSR topics, a predisposition towards trust and support for the particular S/CSR issue about which the company is communicating all positively moderate the effect of S/CSR communication [30, 35, 40]. Also, consumers who arrive at a website using S/CSR related search terms will react stronger to S/CSR information than users arriving through other search terms or channels [11]. There are also national differences in the perceptiveness to S/CSR information. However, Pekkanen et al. [58] argue that these differences can be fully explained by a different distribution of value orientations in the respective countries.

4.3.3. Company Characteristics. As compared to the two previous research streams, the moderating role of company characteristics has received little attention and is only analyzed by two papers in our sample. They find that the for-profit status of a company disseminating S/CSR information diminishes the perceived usefulness of that information (compared to non-profits) [26] and that companies in controversial industrial sectors receive more negative comments for using some S/CSR strategies as compared to companies from non-controversial industries [41].
4.3.4. Medium Characteristics. The medium which we are ultimately interested in are D2C online shops. As we could not find direct empirical evidence on this medium, it is all the more interesting what research says about the role of medium characteristics and their effect on the effectiveness of S/CSR information. However, only one paper compares the effectiveness of S/CSR information across various media types. It finds that medium credibility enhances source credibility and message credibility, and leads to positive consumer responses to the S/CSR communication [60].

\section{Discussion and Outlook}

While we could not find any direct empirical evidence describing the effect of S/CSR information in D2C online shops on consumer attitudes and behavior, our review shows that there are plenty of empirical and conceptual studies conducted in related contexts that can inform our understanding of the described phenomenon. The large number of theories used in the analyzed literature indicates how versatile and complex the role of S/CSR information is. While each theory can contribute to our understanding of the phenomenon at hand, they can have different and even conflicting implications. For example, the theory of planned behavior may suggest that companies should report on what the impact of their S/CSR activities is whereas attribution theory would suggest they focus on why 
they are doing the respective activities. Nudging theory and the theory of bounded behavior may favor simple, visual cues whereas rational-choice based theories may favor more extensive pieces of information. More research is needed to better entangle the contingencies on which the explanatory power of these theories hinges. For the D2C context, these theories can help us form qualified hypotheses about the impact of various types of S/CSR information on consumer attitudes and behavior that can then be tested in future research.

In addition, we can gain some insights from empirical research on S/CSR information in related contexts. In general, extant results can best be described as mixed, i.e. whereas S/CSR information positively influences brand perceptions and purchase intentions in some cases, it can also be perceived negatively in others. O'Rourke and Ringer [11] even warn about a potential bias against green products. Also, media characteristics such as media credibility seem to play an important role [60]. As D2C online shops are a company owned medium, information credibility might be perceived as lower than e.g. the S/CSR ratings on independent review platforms or consumers might attribute profit-oriented motives to the provision of S/CSR information. Thus, transferability of results is not a priori given. However, until more research is conducted in a D2C sphere, the results from other contexts may provide some guidelines as to how S/CSR information should be presented in D2C online shops. In particular, extant results indicate that companies have to know their audience very well and understand what social and environmental issues resonate with them. They should evaluate how high the egoistic or altruistic disposition of their customers is and adapt their communication accordingly. Finally, companies need to understand how company characteristics might moderate the effect of S/CSR information and they should be aware of risks such as greenwashing accusations. Continuously monitoring feedback and behavior changes in response to S/CSR information seems to be a good first step towards a good S/CSR information strategy.

Despite these first findings, several questions and research gaps remain open. First, the number of variables and constructs used in empirical S/CSR research so far is daunting. While frameworks such as the CSR communication model help to structure the field to some extent, a taxonomy structuring all message characteristics and their possible manifestations would help to increase construct clarity and to approach the topic more systematically.

Second, it should be tested to what extent findings regarding the effects of S/CSR information in stores, on corporate websites or on online marketplaces are transferrable to the online D2C context. To this end, research designs from extant studies could be replicated with D2C customers. For example, it could be interesting to analyze whether D2C customers react differently to external (e.g. philanthropic) and internal (e.g. better working conditions) S/CSR initiatives (building upon Amatulli et al. [54]) or to find out what differences exist in direct customers' reactions to S/CSR information as compared to indirect ones (building upon O'Rourke and Ringer [11]).

Third, it is noticeable that most studies in our sample use surveys as data collection method and thus query consumer attitudes rather than behavior. Given the well documented existence of the attitude-behavior gap, this raises the question whether favorable attitudes of D2C customers really translate to higher conversion rates or higher spending. To give a qualified answer to this question, more research with real-world data is needed. Considering the large number of S/CSR oriented D2C online shops and the widespread use of tracking tools, it is very likely that such data already exists. The challenge for research is to get access to such data and to find ways to make it generalizable across various D2C brands and industries. Otherwise, research could aim to create more realistic experimental settings that are closer to tracking purchase decisions rather than just purchase intentions.

Fourth, for scientific research results in this topic to be applied in practice and to create actual impact, we argue that a collection of good practices and design principles that D2C companies can follow is strongly needed. We would thus welcome more design science oriented studies that turn scientific insights into actionable and practically useful advice.

Lastly, the topic at hand has a strong ethical dimension. While "doing good and talking about it" is a widespread and generally accepted business practice, the boundaries between presenting the company in a good light and engaging in greenwashing are often hard to define and need to be discussed. Thus, further research on the business impact of S/CSR information in $\mathrm{D} 2 \mathrm{C}$ online shops should be accompanied by a normative dialogue on the limits of instrumentalizing S/CSR information for positive business outcomes.

\section{Conclusion}

In this literature review we have shown that research on the effect of S/CSR information in a D2C context is sparse, but that theories and empirical evidence from related contexts can help us grasp the issue to some extent. Our contribution is valuable both for research and for practice. For academia, it provides an up-to-date account of the field of consumer-oriented S/CSR communication and demonstrates that the field is researched under many - partly conflicting - 
theoretical lenses, thus indicating a need for further S/CSR specific theory building. For practice, particularly our review of empirical research can serve as an orientation for new D2C companies as well as established brands venturing into D2C. They can refer to the synthesis of these results when deciding how to present S/CSR information in order to produce the optimal combination of social, environmental and business impact.

\section{References}

[1] United Nations, Transforming our world: the 2030 Agenda for Sustainable Development, 2015.

[2] Sustainability, S., and I. Apps, "Sustainability Apps - The Key to Promoting Sustainable Shopping?", Marketing Review St. Gallen(4), 2020, pp. 64-71.

[3] Viciunaite, V., and F. Alfnes, "Informing sustainable business models with a consumer preference perspective", Journal of Cleaner Production 242, 2020, pp. 118417. [4] Go, E., and D.S. Bortree, "What and How to Communicate CSR? The Role of CSR fit, Modality Interactivity, and Message Interactivity on Social Networking Sites", Journal of Promotion Management 23(5), 2017, pp. 727-747.

[5] Dunn, K., and D. Harness, "Communicating corporate social responsibility in a social world: the effects of company-generated and user-generated social media content on CSR attributions and scepticism", Journal of Marketing Management 34(17-18), 2018, pp. 1503-1529.

[6] Reinartz, W., N. Wiegand, and M. Imschloss, "The impact of digital transformation on the retailing value chain", International Journal of Research in Marketing 36(3), 2019, pp. 350-366.

[7] Gielens, K., and J.B.E.M. Steenkamp, "Branding in the era of digital (dis)intermediation", International Journal of Research in Marketing 36(3), 2019, pp. 367-384.

[8] Strähle, J., C. Will, and M. Freise, Communication of Sustainability at European Fashion Online Shops, 2015.

[9] Bartok, O., "The Use of CSR in E-Commerce as a Way to Compete", Journal of Competitiveness 10(4), 2018, pp. 5-20. [10] Gazzola, P., G. Colombo, R. Pezzetti, and L. Nicolescu, "Consumer Empowerment in the Digital Economy: Availing Sustainable Purchasing Decisions”, Sustainability 9(5), 2017, pp. 693.

[11] O'Rourke, D., and A. Ringer, “The Impact of Sustainability Information on Consumer Decision Making", Journal of Industrial Ecology 20(4), 2016, pp. 882-892. [12] Van Marrewijk, M., "Concepts and Definitions of CSR and Corporate Sustainability: Between Agency and Communion", Journal of Business Ethics, Springer Netherlands (2003), 95-105.

[13] World Commission on Environment, Report of the World Commission on Environment and Development: Our Common Future, 1987.

[14] Ott, H., R. Wang, and D. Bortree, “Communicating Sustainability Online: An Examination of Corporate, Nonprofit, and University Websites", Mass Communication and Society 19(5), 2016, pp. 671-687.

[15] Alniacik, U., E. Alniacik, and N. Genc, "How corporate social responsibility information influences stakeholders' intentions", Corporate Social Responsibility and

Environmental Management 18(4), 2011, pp. 234-245.

[16] Spryker, and Die Post, How does D2C become a key profit driver?, 2020.

[17] eMarketer, "US Direct-to-Consumer Ecommerce Sales Will Rise to Nearly \$18 Billion in 2020”, eMarketer Trends, Forecasts \& Statistics.

https://www.emarketer.com/content/us-direct-to-consumerecommerce-sales-will-rise-to-nearly-18-billion-in-2020

[18] Webster, J., and R.T. Watson, "Analyzing the Past to Prepare for the Future: Writing a Literature Review.", MIS Quarterly 26(2), 2002, pp. xiii-xxiii.

[19] vom Brocke, J., A. Simons, K. Riemer, B. Niehaves, R. Plattfaut, and A. Cleven, "Standing on the shoulders of giants: Challenges and recommendations of literature search in information systems research", Communications of the Association for Information Systems, 2015.

[20] vom Brocke, J., A. Simons, B. Niehaves, et al.,

"Reconstructing the Giant: On the Importance of Rigour in Documenting the Literature Search Process", ECIS 2009, 2009, pp. 2206-2217.

[21] Schuitema, G., and J.I.M. de Groot, "Green consumerism: The influence of product attributes and values on purchasing intentions", Journal of Consumer Behaviour 14(1), 2015, pp. 57-69.

[22] Seyfang, G., "Shopping to save the planet?: a critical analysis of sustainable consumption policy and practice", ECPR Joint Sessions, (2004).

[23] Sachdeva, S., J. Jordan, and N. Mazar, "Green consumerism: Moral motivations to a sustainable future", Current Opinion in Psychology 6, 2015, 60-65.

[24] Henkel, C., A.-R. Seidler, J. Kranz, and M. Fiedler, "How to Nudge Pro-Environmental Behavior: An Empirical Study”, ECIS 2019 Proceedings, 2019.

[25] Simon, H.A., A Behavioral Model of Rational Choice, 1955.

[26] Watts, S., "Corporate Sustainability Ratings Databases: Maximizing perceived content usefuless”, ICIS 2015

Proceedings, 2015.

[27] Thaler, R.H., and C.R. Sunstein, Nudge, Penguin Books, London, 2009.

[28] Meske, C., and I. Amojo, "Ethical Guidelines for the Construction of Digital Nudges", Hawaii International Conference on System Sciences 2020 (HICSS-53), 2020. [29] Zhang, Y., "IT Enabled Enviornmentally Friendly Consumption: IT Features Adressing Challenges in Consumer Decision Making”, ECIS 2012 Proceedings, 2012. [30] Sayogo, D., J. Zhang, S. Picazo-Vela, B. Bahaddin, and L. Luna-Reyes, "Understanding the Intention to Trust Product Information and Certifications to Promote Sustainable Consumption: Applying the Theory of Planned Behavior", Hawaii International Conference on System Sciences 2018 (HICSS-51), 2018.

[31] Yang, S., L. Li, and J. Zhang, "Understanding Consumers' Sustainable Consumption Intention at China's Double-11 Online Shopping Festival: An Extended Theory of Planned Behavior Model", Sustainability 10(6), 2018. [32] Ramesh, M., and C. Samudhra Rajkumar, "Purchase decision of online shoppers towards green products", International Journal of Recent Technology and Engineering 
8(3), 2019, pp. 3835-3842.

[33] Lembcke, T.-B., N. Engelbrecht, M. Willnat, and S. Lichtenberg, "Behavioral Design in Online Supermarkets: How Virtual Shopping Cart Functions Impact Sustainable Consumption", AMCIS 2020 Proceedings, 2020.

[34] Klinglmayr, J., B. Bergmair, M.A. Klaffenböck, L. Hörmann, and E. Pournaras, "Sustainable Consumerism via Context-Aware Shopping”, International Journal of Distributed Systems and Technologies 8(4), 2017, pp. 54-72. [35] Meinke, K., and O. Mustorp, "Understanding how awareness of a fashion company's social sustainability practices influences consumers' perception of brand image, as compared to one's interest in fashion", Marketing and Consumption, 2017.

[36] Vermeir, I., and W. Verbeke, "Sustainable food consumption: Exploring the consumer 'attitude - Behavioral intention' gap", Journal of Agricultural and Environmental Ethics 19(2), 2006, pp. 169-194.

[37] Ramesh, K., R. Saha, S. Goswami, Sekar, and R. Dahiya, "Consumer's response to CSR activities: Mediating role of brand image and brand attitude", Corporate Social Responsibility and Environmental Management 26(2), 2019, pp. 377-387.

[38] Carrington, M.J., B.A. Neville, and G.J. Whitwell, "Lost in translation: Exploring the ethical consumer intentionbehavior gap", Journal of Business Research 67(1), 2014, pp. 2759-2767.

[39] Kotler, P., K.L. Keller, M. Brady, M. Goodman, and T. Hansen, Marketing Management, Pearson, 2016.

[40] Pérez, A., M. del Mar García de los Salmones, and M.T. Liu, "Maximising business returns to corporate social responsibility communication: An empirical test", Business Ethics 28(3), 2019, pp. 275-289.

[41] Song, B., and J. Wen, "Online corporate social responsibility communication strategies and stakeholder engagements: A comparison of controversial versus noncontroversial industries", Corporate Social Responsibility and Environmental Management 27(2), 2020, pp. 881-896. [42] Becker-Olsen, K.L., B.A. Cudmore, and R.P. Hill, "The impact of perceived corporate social responsibility on consumer behavior", Journal of Business Research 59(1), 2006, pp. 46-53.

[43] Du, S., C.B. Bhattacharya, and S. Sen, "Maximizing Business Returns to Corporate Social Responsibility (CSR):

The Role of CSR Communication", International Journal of Management Reviews 12(1), 2010, pp. 8-19.

[44] Selamat, M.H., R.M. Saat, and R.H. Raja Mohd Ali, "Web site presentation of corporate social responsibility towards customers trust and corporate image", International Journal of Economics and Financial Issues 6(7Special Issue), 2016, pp. 215-224.

[45] Lindenberg, S., and L. Steg, "Normative, gain and hedonic goal frames guiding environmental behavior", Journal of Social Issues 63(1), 2007, pp. 117-137.

[46] Stern, P.C., "New Environmental Theories: Toward a Coherent Theory of Environmentally Significant Behavior", Journal of Social Issues 56(3), 2000, pp. 407-424.

[47] Angeles, R., "Attributes of Consumers Most Likely to Use Goodguide.com Sustainability Information About Green Household Products", MCIS 2014 Proceedings, 2014.
[48] Schaltegger, S., R. Burritt, D. Zvezdov, J. Hörisch, and J. Tingey-Holyoak, "Management Roles and Sustainability Information. Exploring Corporate Practice", Australian Accounting Review 25(4), 2015, pp. 328-345.

[49] Rowbottom, N., and A. Lymer, "Exploring the use of online corporate sustainability information", Accounting Forum 33(2), 2009, pp. 176-186.

[50] Parker, C.M., E. Bellucci, L. Torlina, B. Fraunholz, and A. Zutshi, "Conceptualising how SMEs incorporate green content in their websites", Australasian Journal of Information Systems 18(3), 2014, pp. 137-160.

[51] Demarque, C., L. Charalambides, D.J. Hilton, and L. Waroquier, "Nudging sustainable consumption: The use of descriptive norms to promote a minority behavior in a realistic online shopping environment", Journal of Environmental Psychology 43, 2015, pp. 166-174.

[52] Wang, C., T. Zhu, H. Yao, and Q. Sun, "The impact of green information on the participation intention of consumers in online recycling: An experimental study", Sustainability (Switzerland) 12(6), 2020.

[53] Peifer, J.L., and D.T. Newman, "Making the business case for corporate social responsibility and perceived trustworthiness: A cross-stakeholder analysis", Business and Society Review, 2020.

[54] Amatulli, C., M. De Angelis, D. Korschun, and S. Romani, 'Consumers' perceptions of luxury brands' CSR initiatives: An investigation of the role of status and conspicuous consumption", Journal of Cleaner Production 194, 2018, pp. 277-287.

[55] Cho, Y.-N., R.L. Soster, and S. Burton, "Enhancing Environmentally Conscious Consumption through Standardized Sustainability Information", Journal of Consumer Affairs 52(2), 2018, pp. 393-414.

[56] Chung, S., and S.Y. Lee, "Cognitive processing of corporate social responsibility campaign messages: the effects of emotional visuals on memory", Media Psychology 23(2), 2020, pp. 244-268.

[57] Giannoccaro, G., D. Carlucci, R. Sardaro, L. Roselli, and B.C. De Gennaro, "Assessing consumer preferences for organic vs eco-labelled olive oils", Organic Agriculture 9(4), 2019, pp. 483-494.

[58] Pekkanen, T.-L., S. Pätäri, L. Albadera, and A. Jantunen, "Who Cares About Product Sustainability Information at the Moment of Purchase? Consumer Evidence from Three Countries", Sustainable Development 26(3), 2018, pp. 229-242.

[59] Oates, C., S. McDonald, P. Alevizou, K. Hwang, W. Young, and L. McMorland, "Marketing sustainability: Use of information sources and degrees of voluntary simplicity", Journal of Marketing Communications 14(5), 2008, pp. 351365

[60] Lee, J., and M. Cho, “The Effects of Consumers' Media Exposure, Attention, and Credibility on Pro-environmental Behaviors", Journal of Promotion Management 26(3), 2020, pp. 434-455.

[61] Mohrenfels, H.W. von, and D. Klapper, "The Influence of Mobile Product Information on Brand Perception and Willingness to Pay for Green and Sustainable Products", ICIS 2012 Proceedings, 2012. 